\title{
Trapped ghosts: a new class of wormholes
}

\author{
Kirill A. Bronnikov ${ }^{a, 1}$, and Sergey V. Sushkov ${ }^{b, 2}$ \\ a Center of Gravitation and Fundamental Metrology, VNIIMS, Ozyornaya St. 46, Moscow 117361, Russia; \\ Institute of Gravitation and Cosmology, PFUR, Miklukho-Maklaya St. 6, Moscow 117198, Russia \\ ${ }^{b}$ Department of General Relativity and Gravitation, Kazan State University, \\ Kremlyovskaya St. 18, Kazan 420008, Russia; \\ Department of Mathematics, Tatar State University of Humanities and Education, \\ Tatarstan St. 2, Kazan 420021, Russia
}

\begin{abstract}
We construct examples of static, spherically symmetric wormhole solutions in general relativity with a minimally coupled scalar field $\phi$ whose kinetic energy is negative in a restricted region of space near the throat (of arbitrary size) and positive far from it. Thus in such configurations a "ghost" is trapped in the strong-field region, which may in principle explain why no ghosts are observed under usual conditions. Some properties of general wormhole models with the $\phi$ field are revealed: it is shown that (i) trapped-ghost wormholes are only possible with nonzero potentials $V(\phi)$; (ii) in twice asymptotically flat wormholes, a nontrivial potential $V(\phi)$ has an alternate sign, and (iii) a twice asymptotically flat wormhole which is mirror-symmetric with respect to its throat has necessarily a zero Schwarzschild mass at both asymptotics.
\end{abstract}

PACS: 04.20.-q, 04.20.Jb, 04.40.-b

\section{Introduction}

It is well known that the existence of traversable Lorentzian wormholes as solutions to the equations of general relativity requires "exotic matter", i.e., matter that violates the null energy condition $[1,2]$. In particular, for static, spherically symmetric configurations with a minimally coupled scalar field as a source, wormhole solutions are only possible if the scalar field is phantom, i.e., has a wrong sign of kinetic energy [3-5]. In alternative theories of gravity, such as scalar-tensor, multidimensional and curvature-nonlinear theories, wormholes also turn out to be possible only if some of the degrees of freedom are of phantom nature $[3,6]$ (see also the review [7] and references therein).

Meanwhile, macroscopic phantom matter has not yet been observed, which puts to doubt the very possibility of obtaining realistic wormholes even in a remote future and even by a highly advanced civilization.

In this paper, we would like to discuss an interesting opportunity of obtaining wormhole configurations in general relativity with a kind of matter which possesses phantom properties only in a restricted region of space, somewhere close to the throat, whereas far away from it all standard energy conditions are observed. As an example of such matter, we consider static, spherically symmetric configurations of a minimally coupled scalar field with the Lagrangian ${ }^{3}$

$$
L_{s}=-\frac{1}{2} h(\phi) g^{\mu \nu} \partial_{\mu} \phi \partial_{\nu} \phi-V(\phi),
$$

\footnotetext{
${ }^{1}$ e-mail: kb20@yandex.ru

${ }^{2}$ e-mail: sergey_sushkov@mail.ru

${ }^{3}$ We choose the metric signature $(-,+,+,+)$, the units $c=$ $\hbar=8 \pi G=1$, and the sign of $T_{\mu}^{\nu}$ such that $T_{0}^{0}$ is the energy density.
}

where $h(\phi)$ and $V(\phi)$ are arbitrary functions. If $h(\phi)$ has a variable sign, it cannot be absorbed by re-definition of $\phi$ in its whole range. A case of interest is that $h>0$ (that is, the scalar field is canonical, with positive kinetic energy) in a weak field region and $h<0$ (the scalar field is of phantom, or ghost nature) in some restricted region where a wormhole throat can be expected. In this sense it can be said that the ghost is trapped. A possible transition between $h>0$ and $h<0$ in cosmology was considered in [8].

The paper is organized as follows. In Section 2 we present the basic equations and show why the above strategy cannot be realized for massless fields $(V(\phi) \equiv$ $0)$. In Section 3 we describe some general properties of the system and obtain explicit examples of "trappedghost" wormhole solutions using the inverse-problem method, and Section 4 is a conclusion.

\section{Scalar fields with a variable ki- netic term}

The general static, spherically symmetric metric can be written as

$$
d s^{2}=-\mathrm{e}^{2 \gamma(u)} d t^{2}+\mathrm{e}^{2 \alpha(u)} d u^{2}+\mathrm{e}^{2 \beta(u)} d \Omega^{2} .
$$

where $u$ is an arbitrary radial coordinate and $d \Omega^{2}=$ $\left(d \theta^{2}+\sin ^{2} \theta d \varphi^{2}\right)$ is the linear element on a unit sphere. A scalar field $\phi(u)$ with the Lagrangian (1) in a spacetime with the metric (2) has the stress-energy tensor (SET)

$$
\begin{gathered}
T_{\mu}^{\nu}=\frac{1}{2} h(u) \mathrm{e}^{-2 \alpha} \phi^{\prime}(u)^{2} \operatorname{diag}(1,-1,1,1) \\
+\delta_{\mu}^{\nu} V(u) .
\end{gathered}
$$


(the prime denotes $d / d u$ ). The kinetic energy density is positive if $h(\phi)>0$ and negative if $h(\phi)<0$, so wormhole solutions sought for must be obtained with $h>0$ at large values of the spherical radius $r(u)=\mathrm{e}^{\beta}$ and $h<0$ at smaller radii $r$. One can show, however, that this goal cannot be achieved for a massless field $(V(\phi) \equiv 0)$.

Indeed, in the massless case, the SET (3) has the same structure as for a usual massless scalar field with $h= \pm 1$. Therefore the metric has the same form as in this simple case and should be reduced to the Fisher metric [9] if $h>0$ and to the corresponding solution for a phantom scalar, first found by Bergmann and Leipnik [10] (it is sometimes called "anti-Fisher") in case $h(\phi)<$ 0 . Let us reproduce this solution for our scalar (1) in the simplest joint form, following [3].

Two combinations of the Einstein equations

$$
R_{\mu}^{\nu}-\frac{1}{2} \delta_{\mu}^{\nu} R=-T_{\mu}^{\nu}
$$

for the metric (2) and the SET (3) with $V \equiv 0$ read $R_{0}^{0}=0$ and $R_{0}^{0}+R_{2}^{2}=0$. Choosing the harmonic radial coordinate $u$, such that $\alpha(u)=2 \beta(u)+\gamma(u)$, we easily solve these equations. Indeed, the first of them reads simply $\gamma^{\prime \prime}=0$, while the second one is written as $\beta^{\prime \prime}+\gamma^{\prime \prime}=\mathrm{e}^{2(\beta+\gamma)}$. Solving them, we have

$$
\begin{aligned}
& \gamma=-m u, \\
& \mathrm{e}^{-\beta-\gamma}=s(k, u):= \begin{cases}k^{-1} \sinh k u, & k>0, \\
u, & k=0, \\
k^{-1} \sin k u, & k<0 .\end{cases}
\end{aligned}
$$

where $k$ and $m$ are integration constants; two more integration constants have been suppressed by choosing the zero point of $u$ and the scale along the time axis. As a result, the metric has the form $[3]^{4}$

$$
d s^{2}=-\mathrm{e}^{-2 m u} d t^{2}+\frac{\mathrm{e}^{2 m u}}{s^{2}(k, u)}\left[\frac{d u^{2}}{s^{2}(k, u)}+d \Omega^{2}\right]
$$

(note that spatial infinity here corresponds to $u=0$ and $m$ has the meaning of the Schwarzschild mass). Moreover, with these metric functions, the $\left(\begin{array}{l}1 \\ 1\end{array}\right)$ component of the Einstein equations (4) leads to

$$
k^{2} \operatorname{sign} k=m^{2}+\frac{1}{2} h(\phi) \phi^{\prime 2} .
$$

It means that $h(\phi) \phi^{\prime 2}=$ const, that is, $h(\phi)$ cannot change its sign within a particular solution which is characterized by certain values of the constants $m$ and $k$. The situation remains the same if, instead of a single scalar field, there is a nonlinear sigma model with multiple scalar fields $\phi^{a}$ and the Lagrangian

$$
L_{\sigma}=-\frac{1}{2} h_{a b} g^{\mu \nu} \partial_{\mu} \phi^{a} \partial_{\nu} \phi^{b},
$$

where $h_{a b}$ are functions of $\phi^{a}$ : the metric then has the same form (6), and a relation similar to (7) reads [13]

$$
k^{2} \operatorname{sign} k=m^{2}+\frac{1}{2} h_{a b} \phi^{a \prime} \phi^{b \prime} .
$$

\footnotetext{
${ }^{4}$ For a detailed description of the properties of Fisher and antiFisher solutions see $[5,12,13]$ and references therein.
}

Therefore the quantity that determines the canonical or phantom nature of the scalars, $h_{a b} \phi^{a \prime} \phi^{b \prime}$, is constant. If the matrix $h_{a b}$ is not positive- or negative-definite, some solutions due to (8) can be wormhole while others correspond to a canonical scalar and have a Fisher central singularity [13], but there are no solutions of trapped-ghost character.

Returning to our system with the Lagrangian (1), we can assert that trapped-ghost wormholes can only exist with a nonzero potential $V(\phi)$. To find such configurations, it is helpful to use the so-called quasiglobal gauge $\alpha+\gamma=0$, so that the metric (2) takes the form

$$
d s^{2}=-A(u) d t^{2}+\frac{d u^{2}}{A(u)}+r^{2}(u) d \Omega^{2},
$$

where $A(u)$ is called the redshift function and $r(u)$ the area function. Then the Einstein-scalar equations can be written as

$$
\begin{aligned}
\left(A r^{2} h \phi^{\prime}\right)^{\prime}-\frac{1}{2} A r^{2} h^{\prime} \phi^{\prime} & =r^{2} d V / d \phi, \\
\left(A^{\prime} r^{2}\right)^{\prime} & =-2 r^{2} V ; \\
2 r^{\prime \prime} / r & =-h(\phi){\phi^{\prime}}^{2} ; \\
A\left(r^{2}\right)^{\prime \prime}-r^{2} A^{\prime \prime} & =2, \\
-1+A^{\prime} r r^{\prime}+A r^{\prime 2} & =r^{2}\left(\frac{1}{2} h A \phi^{\prime 2}-V\right),
\end{aligned}
$$

where the prime again denotes $d / d u$. Eq. (10) follows from (11)-(13), which, given the potential $V(\phi)$ and the kinetic function $h(\phi)$, form a determined set of equations for the unknowns $r(u), A(u), \phi(u)$. Eq. (14) (the $\left(\begin{array}{l}1 \\ 1\end{array}\right)$ component of the Einstein equations), free from second-order derivatives, is a first integral of (10)(13) and can be obtained from (11)-(13) by excluding second-order derivatives. Moreover, Eq. (13) can be integrated giving

$$
B^{\prime}(u) \equiv\left(A / r^{2}\right)^{\prime}=2(3 m-u) / r^{4},
$$

where $B(u) \equiv A / r^{2}$ and $m$ is an integration constant equal to the Schwarzschild mass if the metric (2) is asymptotically flat as $u \rightarrow \infty(r \approx u, A=1-2 m / u+$ $o(1 / u))$. If there is a flat asymptotic as $u \rightarrow-\infty$, the Schwarzschild mass there is equal to $-m(r \approx|u|$, $A=1+2 m /|u|+o(1 / u)$.

Thus in any wormhole solution with two flat asymptotics we inevitably have masses of opposite signs, just as is the case in the well-known special solution the anti-Fisher wormhole $[5,11,12]$ whose metric in the gauge (9) reads

$$
d s^{2}=-\mathrm{e}^{-2 m z} d t^{2}+\mathrm{e}^{2 m z}\left[d u^{2}+\left(k^{2}+u^{2}\right) d \Omega^{2}\right],
$$

with $k<0$ and $z=|k|^{-1} \cot ^{-1}(u /|k|)$ [the constants $m$ and $k$ have the same meaning as in (5)-(7)].

It is also clear that $m=0$ in all symmetric solutions to Eqs. (10)-(14), such that $r(u)$ and $A(u)$ are even functions. Indeed, in this case $B^{\prime}(u)$ is odd, hence $m=$ 0 in (15). 


\section{Models with a trapped ghost}

If one specifies the functions $V(\phi)$ and $h(\phi)$ in the Lagrangian (1), it is, in general, hard to solve the above equations. Alternatively, to find examples of solutions possessing some particular properties, one may employ the inverse problem method, choosing some of the functions $r(u), A(u)$ or $\phi(u)$ and then reconstructing the form of $V(\phi)$ and/or $h(\phi)$. We will do so, choosing a function $r(u)$ that describes a wormhole profile. Then $A(u)$ is found from (15) and $V(u)$ from (11). The function $\phi(u)$ is found from (12) provided $h(\phi)$ is known; however, using the scalar field parametrization freedom, we can, vice versa, choose a monotonic function $\phi(u)$ (which will yield an unambiguous function $V(\phi)$ ) and find $h(u)$ from Eq. (12).

Let us discuss what kind of function $r(u)$ is required for our purpose.

1. The wormhole throat ( $u=0$ without loss of generality) is a minimum of $r(u)$, that is,

$$
r(0)=a, \quad r^{\prime}(0)=0, \quad r^{\prime \prime}(0)>0
$$

with $a=$ const $>0$ (these requirements are sometimes called the flare-out conditions). So $r(u)$ must have such a minimum.

2. In a trapped-ghost wormhole, by definition, the kinetic coupling function $h(\phi)$ is negative near the throat and positive far from it. According to (12), this means that $r^{\prime \prime}$ is positive at small $|u|$ and negative at sufficiently large $|u|$.

3. If the wormhole is asymptotically flat at large $|u|$, we should have

$$
r(u) \approx|u| \quad \text { as } \quad u \rightarrow \pm \infty .
$$

A simple example of the function $r(u)$ satisfying the requirements $1-3$ is (see Fig. 1):

$$
r(u)=a \frac{\left(x^{2}+\lambda\right)}{\sqrt{x^{2}+\lambda^{2}}}, \quad \lambda=\text { const }>2 .
$$

where $x=u / a$, and $a$ is the (arbitrary) throat radius.

Now we can integrate Eq. (15). Assuming $m=0$, we find (see Fig. 2)

$$
A(u)=\frac{3 x^{4}+3 \lambda(\lambda+1) x^{2}+\lambda^{2}\left(\lambda^{2}+\lambda+1\right)}{3\left(x^{2}+\lambda\right)\left(x^{2}+\lambda^{2}\right)} .
$$

Substituting the expressions (17) and (18) into (11), we obtain the potential $V$ as a function of $u$ or $x=u / a$ :

$$
V(u)=\frac{\lambda^{2}(\lambda-1)^{2}\left[-6 x^{4}+\lambda(\lambda-5) x^{2}+\lambda^{3}(\lambda+1)\right]}{3 a^{2}\left(x^{2}+\lambda\right)^{2}\left(x^{2}+\lambda^{2}\right)^{3}} .
$$

One can notice that $V(u)<0$ at large $|u|$. The negative sign of the potential in a certain range of $u$ is not

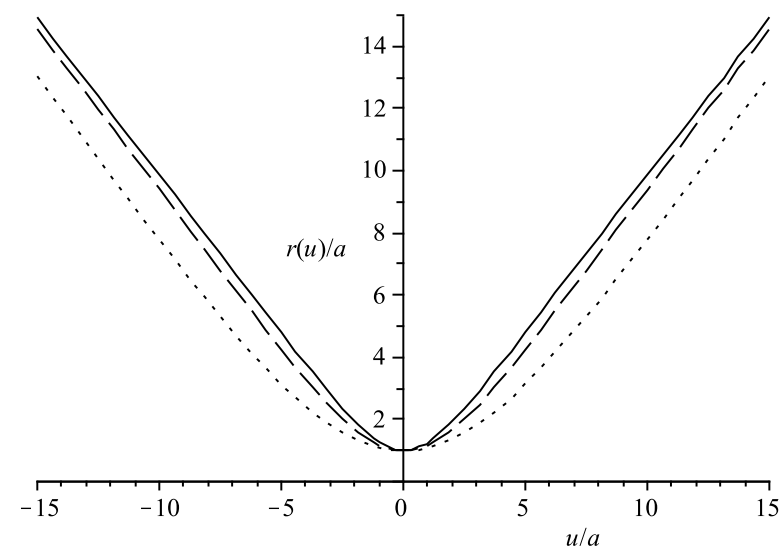

Figure 1: Plots of $r(u) / a$ given by Eq. (17) with $\lambda=$ $3 ; 5 ; 10$ (solid, dashed, and dotted lines, respectively).

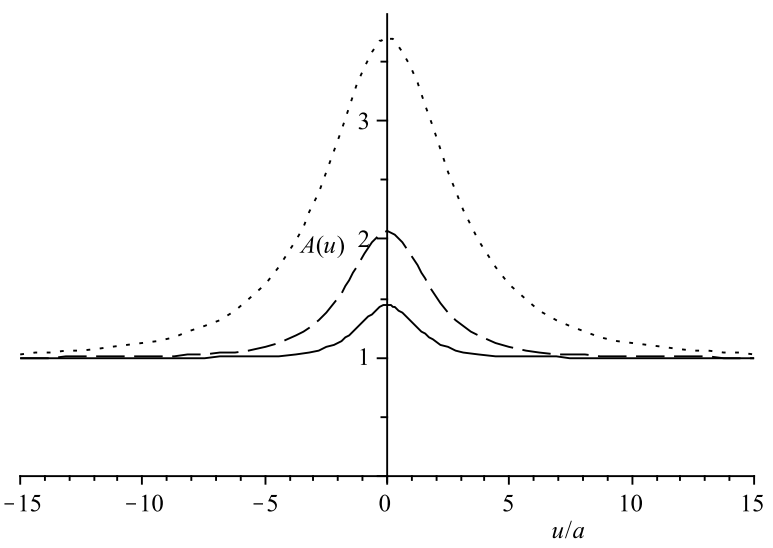

Figure 2: Plots of $A(u)$ given by Eq. (18) with $\lambda=$ $3 ; 5 ; 10$ (solid, dashed, and dotted lines, respectively).

a shortcoming of this particular model but a direct consequence of the field equations. Indeed, as follows from (15), we have $A^{\prime} r^{2} \underset{u \rightarrow \pm \infty}{\longrightarrow} 2 m$ at both flat aymptotics. Consequently, due to (11),

$$
\int_{-\infty}^{+\infty} r^{2} V(u) d u=0
$$

so that if $V(u) \not \equiv 0$, it has an alternate sign.

To construct $V$ as an unambiguous function of $\phi$ and to find $h(\phi)$, it makes sense to choose a monotonic function $\phi(u)$. It is convenient to assume

$$
\phi(u)=\frac{2 \phi_{0}}{\pi} \arctan \frac{x}{\lambda}, \quad \phi_{0}=\frac{\pi a}{2} \sqrt{\frac{2(\lambda-2)}{\lambda}},
$$

and $\phi$ has a finite range: $\phi \in\left(-\phi_{0}, \phi_{0}\right)$, which is common to kink configurations. Thus we have $x=u / a=$ $\lambda \tan \left(\pi \phi / 2 \phi_{0}\right)$, whose substitution into Eq. (19) gives an expression for $V(\phi)$ defined in this finite range. The function $V(\phi)$ can be extended to the whole real axis, $\phi \in \mathbb{R}$, by supposing $V(\phi) \equiv 0$ at $|\phi| \geq \phi_{0}$. Plots of the extended potential $V(\phi)$ are shown in Fig. 3. 


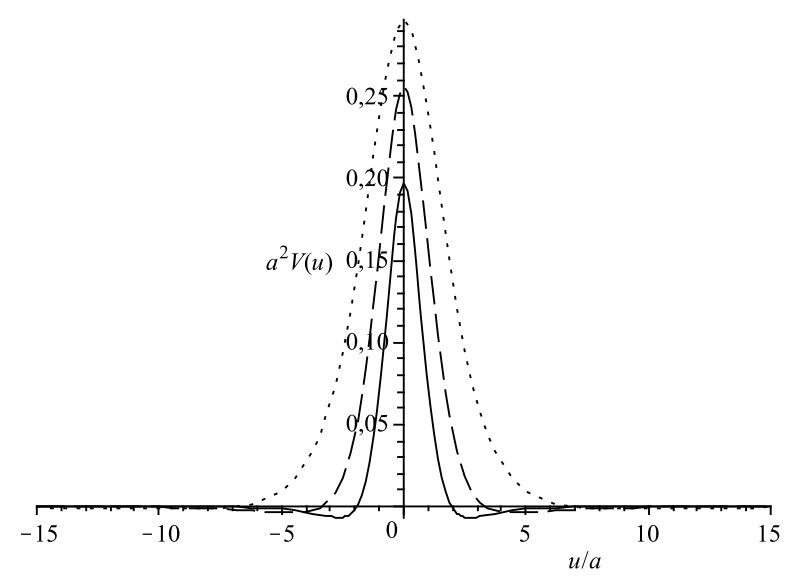

Figure 3: Plots of $V(\phi)$ given by Eq. (19) with $\lambda=$ $3 ; 5 ; 10$ (solid, dashed, and dotted lines, respectively).

The expression for $h(\phi)$ is found from (12) as follows:

$$
h(\phi)=\frac{(\lambda-2) x^{2}+\lambda^{2}(1-2 \lambda)}{a^{2}(\lambda-2)\left(x^{2}+\lambda\right)},
$$

where $x=\lambda \tan \left(\pi \phi / 2 \phi_{0}\right)$. The function $h(\phi)$ given by Eq. (21) is also defined in the interval $\left(-\phi_{0}, \phi_{0}\right)$ and can be extended to $\mathbb{R}$ by supposing $h(\phi) \equiv 1$ at $|\phi| \geq \phi_{0}$. The extended kinetic coupling function $h(\phi)$ is plotted in Fig. 4.

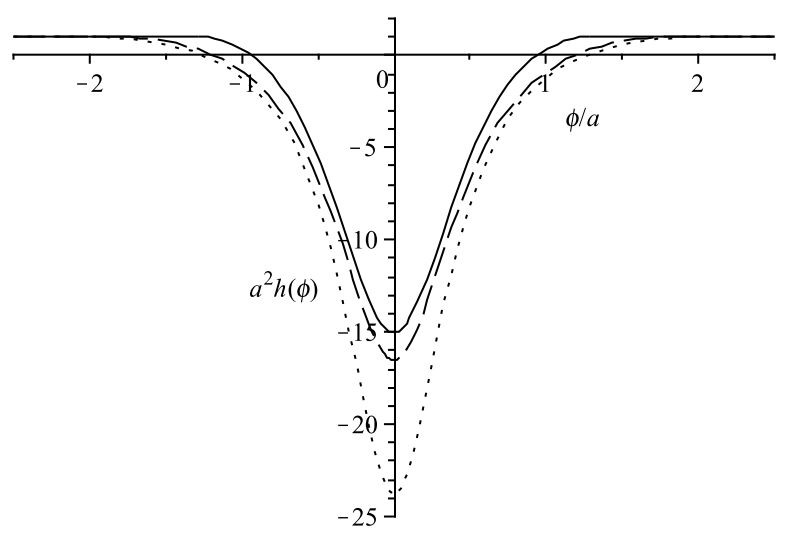

Figure 4: Plots of $h(\phi)$ given by Eq. (21) with $\lambda=$ $3 ; 5 ; 10$ (solid, dashed, and dotted lines, respectively).

It is well known that the null energy condition (NEC) holds for a canonical scalar field and is violated for a phantom one. In our case, it happens for $h(\phi)>0$ and $h(\phi)<0$, respectively. Let us illustrate this using our solution (17), (18), (20) as an example. The NEC reads $-T_{\mu \nu} k^{\mu} k^{\nu} \geq 0$, where $k^{\mu}$ is an arbitrary null vector. Due to the Einstein equations (4), it can be equivalently written as $G_{\mu \nu} k^{\mu} k^{\nu} \geq 0$. Taking the radial null vector $k^{\mu}=\left(A^{-1 / 2}, A^{1 / 2}, 0,0\right)$ in the metric (9) and denoting $\Xi:=G_{\mu \nu} k^{\mu} k^{\nu}$, we find

$$
\Xi(u)=-2 \frac{r^{\prime \prime}}{r}=\frac{2 \lambda\left[x^{2}(\lambda-2)-\lambda^{2}(2 \lambda-1)\right]}{a^{2}\left(x^{2}+\lambda^{2}\right)\left(x^{2}+\lambda\right)},
$$

i.e., it is a multiple of $h(\phi)$ with a positive factor. The plot of $h(\phi)$ thus completely characterizes NEC violation taking place near the throat.

\section{Conclusion}

We have shown that a minimally coupled scalar field may change its nature from canonical to ghost in a smooth way without creating any space-time singularities. This feature, in particular, allows for construction of static, spherically symmetric wormhole models (trapped-ghost wormholes) where the ghost is present in some restricted region around the throat (of arbitrary size) whereas in the weak-field region far from it the scalar has usual canonical properties. One can speculate that if such ghosts do exist in Nature, they are all confined to strong-field regions ("all genies are sitting in their bottles"), but just one of them, having been released, has occupied the whole Universe and plays the part of dark energy (if dark energy is really phantom, which is more or less likely but not certain).

We have also found some general properties of static, spherically symmetric wormhole models in the Einsteinscalar field system under consideration:

(i) trapped-ghost wormholes are only possible with nonzero potentials $V(\phi)$;

(ii) in all wormholes with two flat asymptoyics, $V(\phi)$ has an alternate sign (unless $V \equiv 0$ );

(iii) in any wormhole with two flat asymptoyics, if the Schwarzschild mass equals $m$ at one of them, it equals $-m$ at the other. Hence mirror symmetry with respect to the throat (i.e., the metric functions are even in the radial coordinate $u$ ) implies $m=0$.

\section{Acknowledgments}

This work was supported in part by the Russian Foundation for Basic Research grants No. 08-02-91307, 0802-00325, and 09-02-00677a.

\section{References}

[1] M.S. Morris and K.S. Thorne, Am. J. Phys. 56, 395 (1988).

[2] D. Hochberg and M. Visser, Phys. Rev. D 56, 4745 (1997).

[3] K.A. Bronnikov, Acta Phys. Pol. B 4, 251 (1973).

[4] K.A. Bronnikov, Phys. Rev. D 64, 064013 (2001); grqc/0104092.

[5] S.V. Sushkov and Y.-Z. Zhang, Phys. Rev. D 77, 024042 (2008).

[6] K.A. Bronnikov and A.A. Starobinsky, JETP Lett. 85, 1 (2007). 
[7] F.S.N. Lobo, in: Classical and Quantum Gravity Research, p. 1-78 (Nova Sci. Pub., 2008); ArXiv: 0710.4474 .

[8] H. Kroger, G. Melkonian and S.G. Rubin, Gen. Rel. Grav. 36, 1649 (2004); astro-ph/0310182.

[9] I.Z. Fisher, Zh. Eksp. Teor. Fiz. 18, 636 (1948); grqc/9911008.

[10] O. Bergmann and R. Leipnik, Phys. Rev. 107, 1157 (1957).

[11] H. Ellis, J. Math. Phys. 14, 104 (1973).

[12] K.A. Bronnikov, M.S. Chernakova, J.C. Fabris, N. Pinto-Neto and M.E. Rodrigues, Int. J. Mod. Phys. D 17, 25-42 (2008); gr-qc/0609084.

[13] K.A. Bronnikov, S.V. Chervon, and S.V. Sushkov, Grav. Cosmol. 15, 241 (2009); ArXiv: 0905.3804. 